\title{
Subsídios estruturais à caracterização do sistema caulinar em Cyperaceae
}

\author{
MARIA EMÍLIA MARANHÃO ESTELITAA ${ }^{1,3}$ e ANA CLAUDIA RODRIGUES ${ }^{2}$
}

(recebido: 6 de abril de 2006; aceito: 21 de junho de 2007)

\begin{abstract}
Structural subsidies to stem system caracterization in Cyperaceae). Differential anatomical characters between rhizome and scape are point out. Besides the apical meristems activity in both, rhizome and scape, it was observed the presence and activity of MEP (primary thickening meristem) in the stem; the vascular bundles are of mixed origin (from the procambium and from MEP). In the scape, MEP is not present, and the vascular bundles are procambial. However, strong activity of the intercallary meristem was observed, causing the internode elongation.
\end{abstract}

Key words - anatomy, Cyperaceae, rhizome, scape

RESUMO - (Subsídios estruturais à caracterização do sistema caulinar em Cyperaceae). São enfocados caracteres anatômicos diferenciais entre rizoma e escapo. Além da atividade dos meristemas apicais de ambos, no caule observou-se a presença e atividade do MEP (meristema de espessamento primário); os feixes vasculares, portanto, têm origem mista (do procâmbio e do MEP). No escapo, não ocorre MEP, portanto, os feixes são apenas de origem procambial. Entretanto, observou-se considerável atividade do meristema intercalar, o que produz o alongamento dos entrenós.

Palavras-chave - anatomia, Cyperaceae, escapo, rizoma

\section{Introdução}

As Cyperaceae são plantas cosmopolitas, de hábito herbáceo na grande maioria e que crescem em regiões alagadas ou imediações (Dahlgren et al. 1985). Nos sistemas vegetativos são encontrados diferentes tipos de caules, como por exemplo, tubérculos e bulbos (Wills \& Briscoe 1970) e rizomas (Metcalfe 1971). Vários autores mencionam, ainda, o colmo como mais um tipo de caule na família (Kukkonen 1967, Shepherd 1976, Ueno et al. 1989, Simpson 1993, Bruhl \& Perry 1995, Guaglianone et al. 1998). Entretanto, constata-se que o colmo, neste grupo, deve ser o escapo (Estelita-Teixeira \& Handro 1987). Metcalfe (1971) estudando as Cyperaceae considera como sinônimos escapo e caule aéreo. Ainda, Font-Quer (1975) chama a atenção para a inadequada denominação de colmo nas Cyperaceae. Essas observações podem ser decorrentes de o escapo das Cyperaceae ter longa duração, pois além de ser o principal órgão fotossintetizante, em vários casos apresenta nós com inúmeras brácteas também verdes, conspícuas, semelhantes às folhas, como em espécies de Cyperus e de Rhynchospora.

\footnotetext{
1. Universidade de São Paulo, Instituto de Biociências, Departamento de Botânica, Caixa Postal 11461, 05422-970 São Paulo, SP, Brasil.

2. Universidade Federal de Santa Catarina, Centro de Ciências Biológicas, Departamento de Botânica, 88010-970 Florianópolis, SC, Brasil.

3. Autor para correspondência: estelita7@terra.com.br
}

Poucos autores referem-se corretamente à estrutura em questão, como Wills \& Briscoe (1970) que a chamam de raque em espécies de Cyperus e Estelita-Teixeira \& Handro (1987) que se referem a escapo ao analisarem espécies de Cyperus e Fimbristylis. Entretanto, são denominações baseadas apenas na morfologia externa; as características anatômicas não foram enfocadas.

O objetivo deste estudo foi informar, através dos caracteres morfo-anatômicos, qual órgão é o caule propriamente dito e qual é o escapo; também procurouse determinar os padrões anatômicos dos mesmos.

\section{Material e métodos}

Foram analisadas as seguintes espécies: Cyperus corymbosus Rottb. (SPF 149.815), C. giganteus Vahl. (SPF 138.170), C. hermafroditus (Jacq.) Standl. (SPF 132.154), $C$. papyrus L. (SPF 46.107), Fimbristylis autunnalis (L.) Roem. \& Schult. (SPF 162.660), Fuirena umbellata Rottb. (SPF 158.206), Killinga peruviana Lamark (SPF 21.227) e $K$. brevifolia Rooth (SPF 165.235).

Cyperus giganteus foi analisado mediante cortes seriados do rizoma e do escapo; nas demais amostras foram analisados os órgãos adultos. Fragmentos foram fixados em FAA em álcool 70\% (Johansen 1940) por aproximadamente 70 horas e estocados em etanol 70\%. Procedeu-se à desidratação em álcool butanol terciário e à inclusão em parafina. $\mathrm{O}$ material foi corado com safranina $1 \%$ em álcool $96 \%$ e azul de astra 0,5\% em ácido tartárico 2\% (Bukatsch 1972) e montados em bálsamo-do-canadá. A documentação foi realizada em microscópio Olympus/Vanox. 


\section{Resultados}

As espécies analisadas apresentam rizoma simpodial, contraído, com entrenós muito curtos, do qual partem raízes adventícias (figuras 1-7). Em cada segmento do rizoma o meristema apical vegetativo é protegido por inúmeros catafilos; em condições adequadas, esse meristema transforma-se em reprodutivo à medida que a extremidade do rizoma se posiciona verticalmente. Essa mudança ocorre também nas demais estruturas de proteção, agora denominadas brácteas (figuras 1-7).

Cada escapo pode conter apenas um entrenó, consideravelmente conspícuo, antes de se ramificar, como em Fimbristylis autunnalis (figuras 1-2), Cyperus
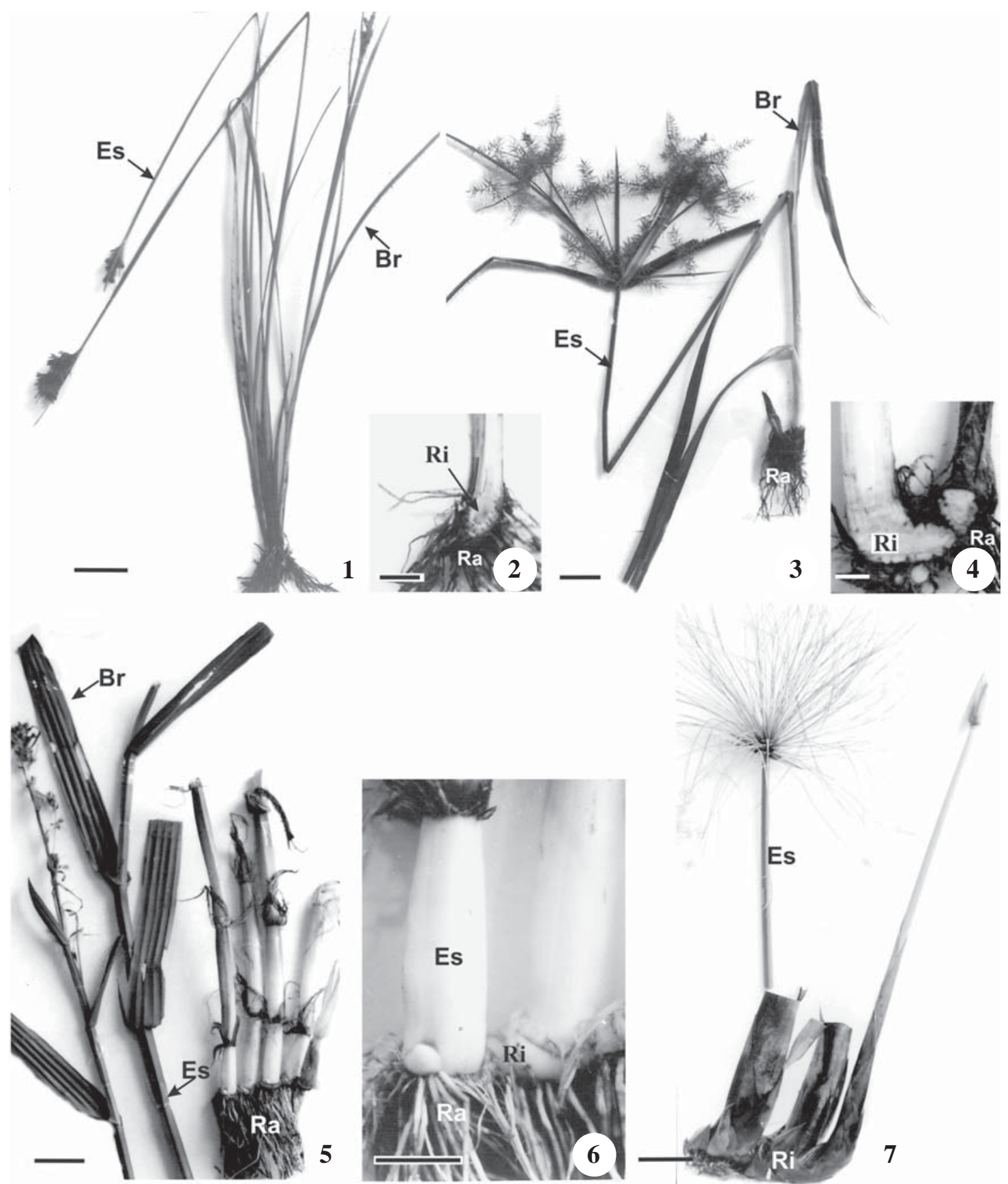

Figuras 1-7. Rizomas e escapos de Cyperaceae. 1-2. Fimbristylis autunnales. 3-4. Cyperus hermafroditus. 5-6. Fuirena umbelata. 7. Cyperus papirus. $(\mathrm{Br}=$ bráctea; $\mathrm{Es}=$ escapo; $\mathrm{Ra}=$ raízes adventícias; $\mathrm{Ri}=$ rizoma). $\mathrm{Barras}=1 \mathrm{~cm}(2,4), 4 \mathrm{~cm}(1,3), 2 \mathrm{~cm}(6), 5 \mathrm{~cm}(5,7)$.

Figures 1-7. Rhizomes and scapes of Cyperaceae. 1-2. Fimbristylis autunnales. 3-4. Cyperus hermafroditus. 5-6. Fuirena umbelata. 7. Cyperus papirus. $(\mathrm{Br}=$ bract; $\mathrm{Es}=$ scape; $\mathrm{Ra}=$ adventitious roots; $\mathrm{Ri}=$ rhizome $) . \mathrm{Bars}=1 \mathrm{~cm}(2,4), 4 \mathrm{~cm}(1,3), 2 \mathrm{~cm}(6), 5 \mathrm{~cm}(5,7)$. 
giganteus e C. papyrus (figura 7), ou pode conter alguns ou vários entrenós, como em $C$. hermafroditus (figuras 3-4) e Fuirena umbellata (figuras 5-6). Nos nós com ou sem ramificações, também estão presentes brácteas (figuras 3, 5).

Outro caráter morfológico significativo diz respeito às diferenças entre as secções transversais. Todos os rizomas têm secções circulares a elípticas, enquanto que a maioria dos escapos tem secções poligonais, como pode ser observado pelas arestas de $F$. umbellata (figura 5).

O rizoma das Cyperaceae caracteriza-se por ter a estrutura primária proveniente da atividade do procâmbio, da protoderme, do meristema fundamental e do meristema de espessamento primário ou MEP (figuras 8, 9). O MEP delimita a região cortical e está relacionado com a formação de gemas vegetativas (figura 10); constatouse também que quando ocorre mais de uma gema o MEP de uma é contínuo como o MEP da outra (figuras 10-11).

A anatomia de $C$. giganteus mostra que a partir de determinada fase do desenvolvimento do rizoma o ápice vegetativo transforma-se em reprodutivo (figura 20). A análise de níveis sucessivos a partir da região caulinar (figura 12) revela que o MEP vai sendo gradativamente eliminado numa disposição espiralada terminando na base do escapo (figuras 13-14). Portanto, no escapo só são encontrados os feixes provenientes do procâmbio (figuras 15-18), que são colaterais (figura 21) e se dispõem aleatoriamente, sem delimitação da região cortical (figura 22). Paralelamente à diferenciação dos feixes vasculares, diferencia-se o aerênquima por formação esquisógena (figuras 21-22). Nesta fase são facilmente distintos os catafilos e as brácteas (figuras 18-19) sendo que estas últimas apresentam parênquima fotossintetizante. Em corte longitudinal do escapo, observa-se intensa atividade do meristema intercalar (figura 20).

Essas características do rizoma são semelhantes para todas as espécies analisadas, como em $K$. peruviana (figuras 24-25), e F. umbellata (figuras 26-27), ou seja, no caule, além da atividade dos meristemas apicais, encontra-se atividade de um meristema lateral. O escapo não apresenta o MEP, mas, o meristema intercalar é conspícuo e com intensa atividade, o que promove um considerável alongamento do entrenó.

\section{Discussão}

A distinção morfológica entre caule propriamente dito e escapo floral é acentuada na grande maioria das espécies, pois os escapos, além de portarem flores, geralmente são efêmeros. Nas monocotiledôneas vários
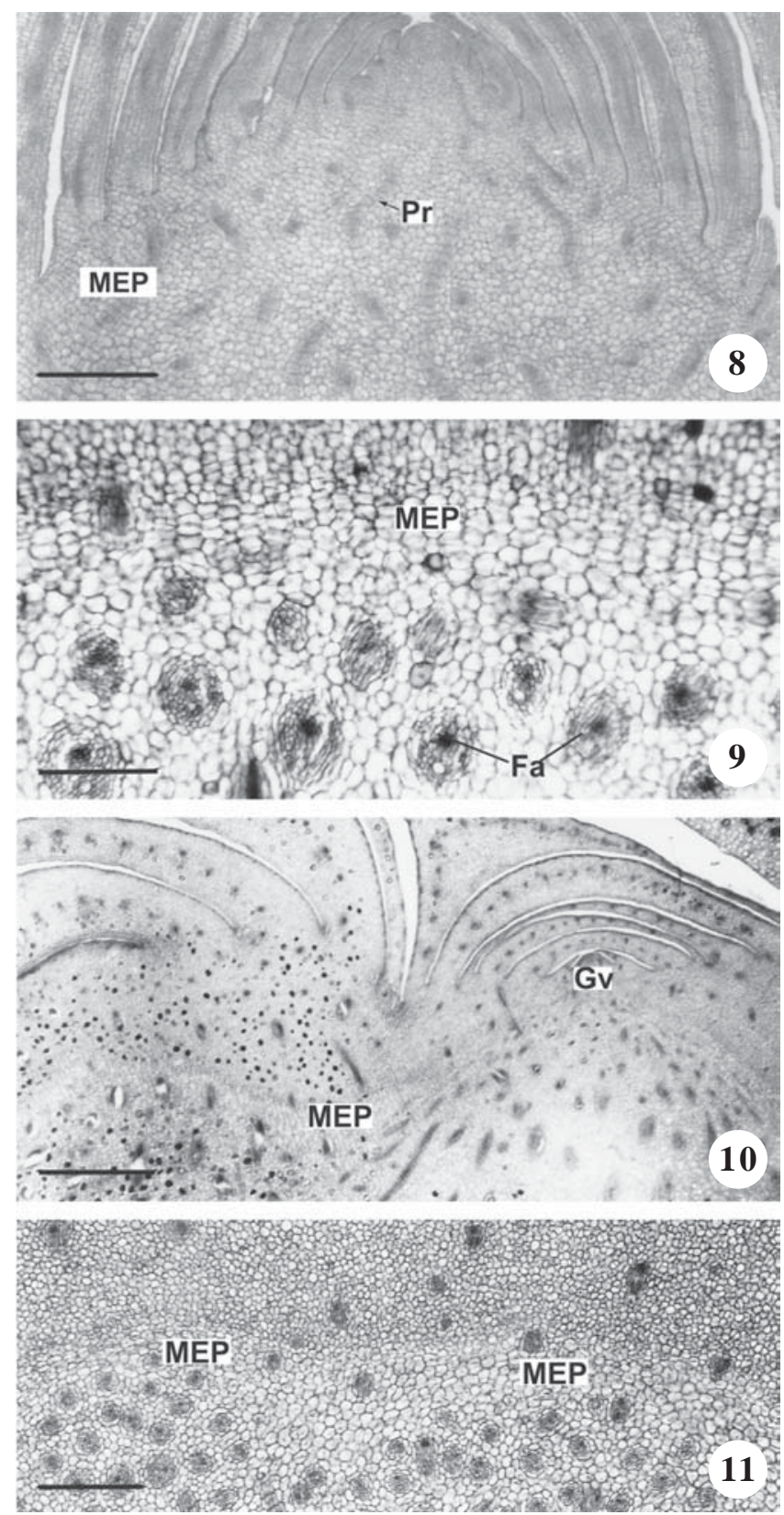

Figuras 8-11. Cyperus giganteus evidenciando o meristema de espessamento primário (MEP). 8. Corte longitudinal do ápice vegetativo. 9-11. Cortes transversais. 9. MEP formando feixes anfivasais. 10. Gema lateral e continuidade do MEP. 11. Detalhe da continuidade do MEP entre rizoma e gema lateral. ( $\mathrm{Fa}=$ feixe vascular anfivasal; $\mathrm{Gv}=$ gema lateral; $\mathrm{Pr}=$ procâmbio). Barras $=500 \mu \mathrm{m}(8,10), 100 \mu \mathrm{m}(9), 200 \mu \mathrm{m}(11)$.

Figures 8-11. Cyperus giganteus showing the primary thickening meristem (MEP) in the rhizome. 8. Longitudinal section of the vegetative apex. 9-11. Transversal sections. 9. Vascular bundles originated by MEP between the rhizome and the lateral bud. ( $\mathrm{Fa}=$ amphivasal vascular bundle; $\mathrm{Gv}=$ lateral bud; $\operatorname{Pr}=$ procambium $).$ Bars $=500 \mu \mathrm{m}(8,10), 100 \mu \mathrm{m}(9)$, 200um (11). 

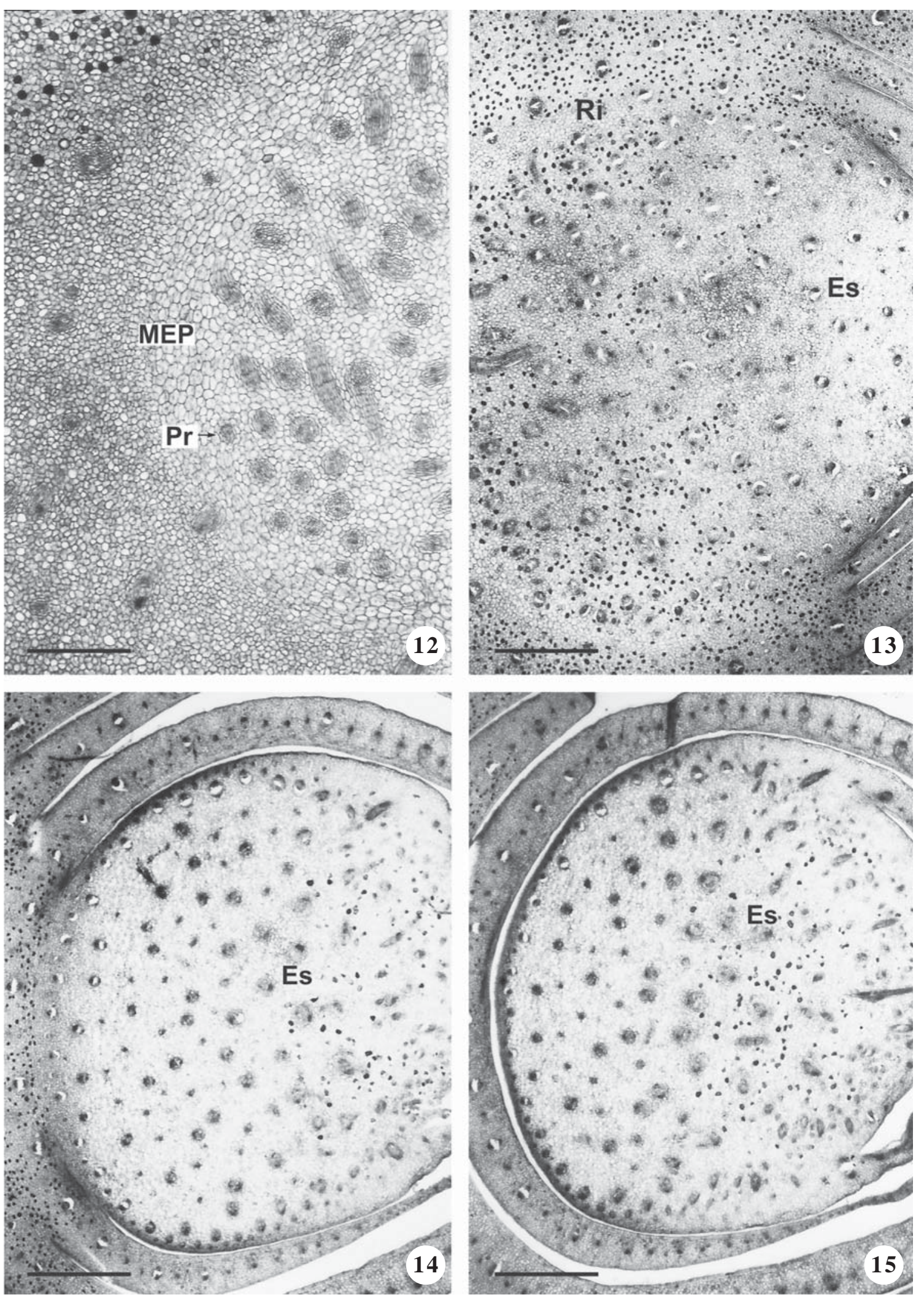

Figuras 12-15. Cyperus giganteus em cortes transversais ascendentes. 12. Rizoma. 13. Região entre rizoma e escapo. 14-15. Escapo. $(E s=$ escapo; $\mathrm{MEP}=$ meristema de espessamento primário; $\mathrm{Pr}=$ procâmbio; $\mathrm{Ri}=$ rizoma). $\mathrm{Barras}=200 \mu \mathrm{m}(12), 500 \mu \mathrm{m}(13-15)$.

Figures 12-15. Ascending transversal sections of Cyperus giganteus. 12. Rhizome. 13. Region between the rhizome and the scape. 14-15. Scape. $($ Es $=$ scape; $M E P=$ primary thickening meristem; $\mathrm{Pr}=$ procambium; $\mathrm{Ri}=$ rhizome $)$. Bars $=200 \mu \mathrm{m}(12), 500 \mu \mathrm{m}(13-15)$. 

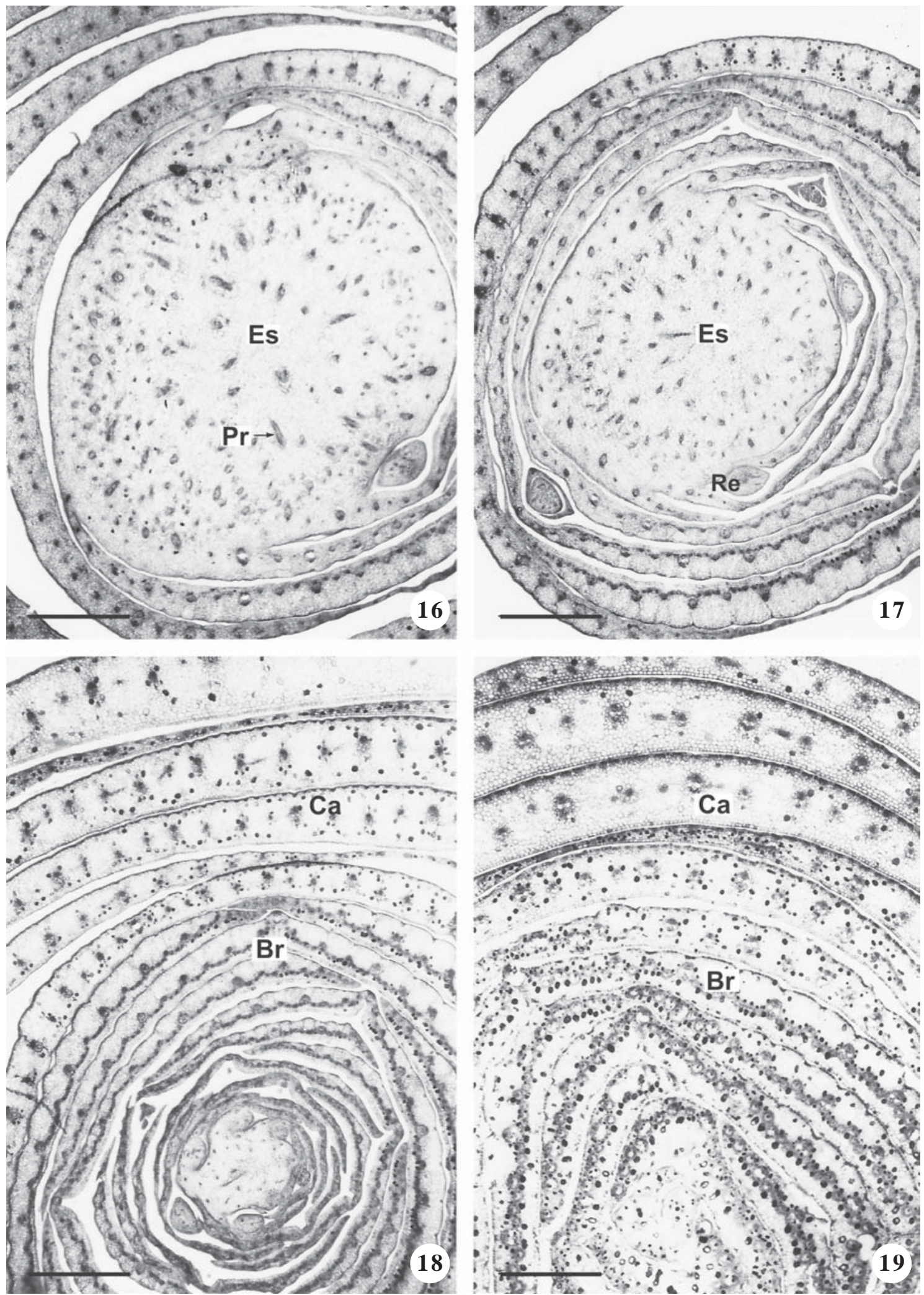

Figuras 16-19. Cyperus giganteus em cortes transversais ascendentes do escapo. $(\mathrm{Br}=$ bráctea; $\mathrm{Ca}=$ catafilo; $\mathrm{Es}=\mathrm{escapo} ; \mathrm{Pr}=$ procâmbio; $\operatorname{Re}=$ ramificação do escapo). Barras $=500 \mu \mathrm{m}(16-19)$.

Figures 16-19. Scape of Cyperus giganteus in ascending transversal section. $(\mathrm{Br}=$ bract; $\mathrm{Ca}=$ cataphyll; $\mathrm{Es}=\mathrm{scape} ; \mathrm{Pr}=$ procambium; $\mathrm{Re}=$ branching of scape $)$. Bars $=500 \mu \mathrm{m}(16-19)$. 

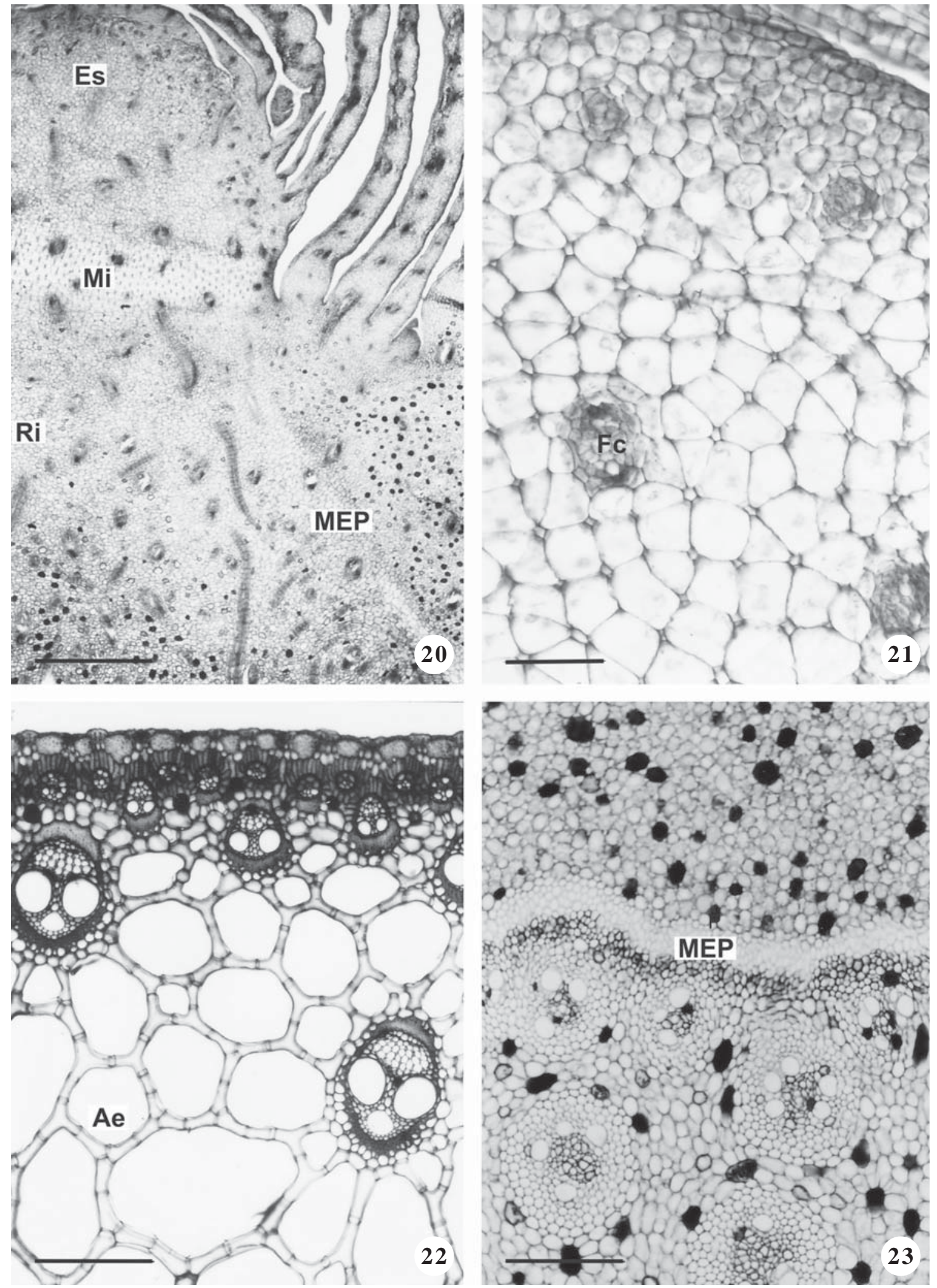

Figuras 20-23. Cyperus giganteus. 20. Secção longitudinal. Escapo (procâmbio e meristema intercalar) e rizoma (procâmbio e meristema de espessamento primário). 21-23. Secções transversais. 21-22. Escapo mostrando diferenciação dos feixes colaterias a partir do procâmbio. 23. Rizoma mostrando a diferenciação dos feixes anfivasais a partir do meristema de espessamento primário. $(\mathrm{Ae}=$ aerênquima; $\mathrm{Es}=$ escapo $; \mathrm{Fc}=$ feixe colateral $; \mathrm{MEP}=$ meristema de espessamento primário; $\mathrm{Mi}=$ meristema intercalar; $\mathrm{Ri}=$ rizoma). Barras $=500 \mu \mathrm{m}(20,22), 50 \mu \mathrm{m}(21), 100 \mu \mathrm{m}(23)$. 
grupos têm o escapo mais conspícuo que o caule, muitas vezes fotossintetizante e de longa duração, como é o caso das Cyperaceae.

Sabe-se que a inflorescência é o resultado de modificações de caules ramificados, portanto, devem apresentar, em cada nó, brácteas ou folhas modificadas. Estas modificações são aplicadas também às flores que, segundo a teoria clássica de Eames (1961), são constituídas por um receptáculo, que corresponde ao eixo caulinar e por estruturas florais que correspondem aos apêndices foliares. Autores como Esau (1965) mencionam que a inflorescência é o resultado da modificação de caules ramificados; portanto apresentam, em cada nó, brácteas ou folhas modificadas. O conjunto rizoma e escapo forma o sistema caulinar em que o último pode ser denominado também de caule reprodutivo. Por razões como estas, é conhecido que o escapo tem estrutura caulinar; entretanto, sempre ocorrem diferenças entre a estrutura morfológica, anatômica e fisiológica de ambos.

As espécies analisadas têm rizomas simpodiais nos quais cada unidade do sistema é contínua com os escapos. Estas características foram também observadas por Rudall (1984) nas Iridaceae. A continuidade entre caule e escapo caracteriza o tipo de florescimento hapaxântico, segundo a classificação adotada por Tomlinson (1970).

Relevante na diferença entre caule e escapo é a presença do MEP só no primeiro, como foi verificado anteriormente por De Mason (1979 a, b) em Allium cepa L. De maneira semelhante Stevenson \& Fisher (1980) ao analisarem Cordyline terminalis Hook verificaram parada da diferenciação do MEP por início da formação da inflorescência; desse modo, admitem existir relação entre o início da inflorescência e o cessar do MEP. Como consequiência, nas espécies que apresentam crescimento secundário, como C. terminalis, Stevenson \& Fisher (1980) constataram que o mesmo nunca ocorre no eixo da inflorescência. Também Rudall (1984) cita que o MES é ausente nos eixos das inflorescências das Iridaceae com crescimento secundário.

Devido à ocorrência do MEP, somente os caules apresentam regiões distintas entre córtex e cilindro vascular, como se pode observar em Heliconia angusta Vell. (Simão \& Scatena 2001), Cyperus giganteus Vahl. (Rodrigues \& Estelita 2002), Canna edulis Ker-Gauler
(Alonso \& Moraes-Dallaqua 2004), em Tillandsia (Segecin \& Scatena 2004) e em várias outras monocotiledôneas (Menezes et al. 2005). Também o padrão anatômico do escapo do presente estudo é semelhante ao encontrado pelos autores acima referidos, no qual se destaca a ausência do MEP, também referida por Stevenson \& Fisher (1980).

$\mathrm{O}$ meristema intercalar é freqüente em folhas, entrenós de muitas monocotiledôneas, escapos e pedicelos. Entretanto, é conhecida a sua maior atividade nos escapos (Sachs 1968) quando comparada com aquela dos caules propriamente ditos. Isso torna fácil o reconhecimento da transformação do ápice vegetativo em floral, pois, entre outros caracteres, ocorre considerável alongamento do entrenó devido à intensa atividade daquele meristema.

De acordo com o aqui exposto, o "colmo" de Eleocharis acuta R. Br., referido por Evans (1965), é o escapo floral. Semelhantes são as ilustrações de dezessete espécies de Uncinia descritas por Kukkonen (1967) e as dezessete espécies de gêneros de Cyperaceae apresentadas por Metcalfe (1971). Também Munro \& Linder (1998) consideram os dois órgãos em questão como pertencentes ao sistema rizoma-colmo. Entretanto, de acordo com o aqui analisado, não existe o caule tipo "colmo" em Cyperaceae; o suposto colmo é sempre um escapo.

Em decorrência dessa análise pode-se afirmar que as "folhas verdes" encontradas nas espécies analisadas são brácteas fotossintetizantes. E ainda, que entre elas observa-se um abrupto alongamento do entrenó, característico do escapo, como salientam Zimmermann \& Tomlinson (1969).

Algumas Cyperaceae, entretanto, podem apresentar parte da região vertical com estrutura caulinar (onde as folhas se dispõem em entrenós mais curtos) e parte com estrutura de escapo, como em Remirea maritima Aubl., segundo ilustrações de Oteng-Yeboah (1975) e de Estelita (1993) onde são observados a presença e os produtos do MEP.

Com o exposto verifica-se também que o escapo tem estrutura mais simples que o rizoma, pois todo o sistema vascular é formado apenas por um meristema, enquanto o rizoma tem o sistema vascular proveniente de dois meristemas. Também no escapo, os feixes

Figures 20-23. Cyperus giganteus. 20. Longitudinal section. Scape (procambium and intercalary meristem) and rhizome (procambium and primary thickening meristem). 21-23. Transversal sections. 21-22. Scape showing the differentiation of the colateral bundle originated by the procambium. 23. Rhizome showing the differentiation of the amphivasal bundles originated by the primary thickening meristem. $(\mathrm{Ae}=$ aerenchyma; $\mathrm{Es}=\mathrm{scape} ; \mathrm{Fc}=$ colateral bundle; $\mathrm{MEP}=$ primary thickening meristem; $\mathrm{Mi}=$ intercalary meristem; $\mathrm{Ri}=$ rhizome $).$ Bars $=500 \mu \mathrm{m}(20,22), 50 \mu \mathrm{m}(21), 100 \mu \mathrm{m}(23)$. 

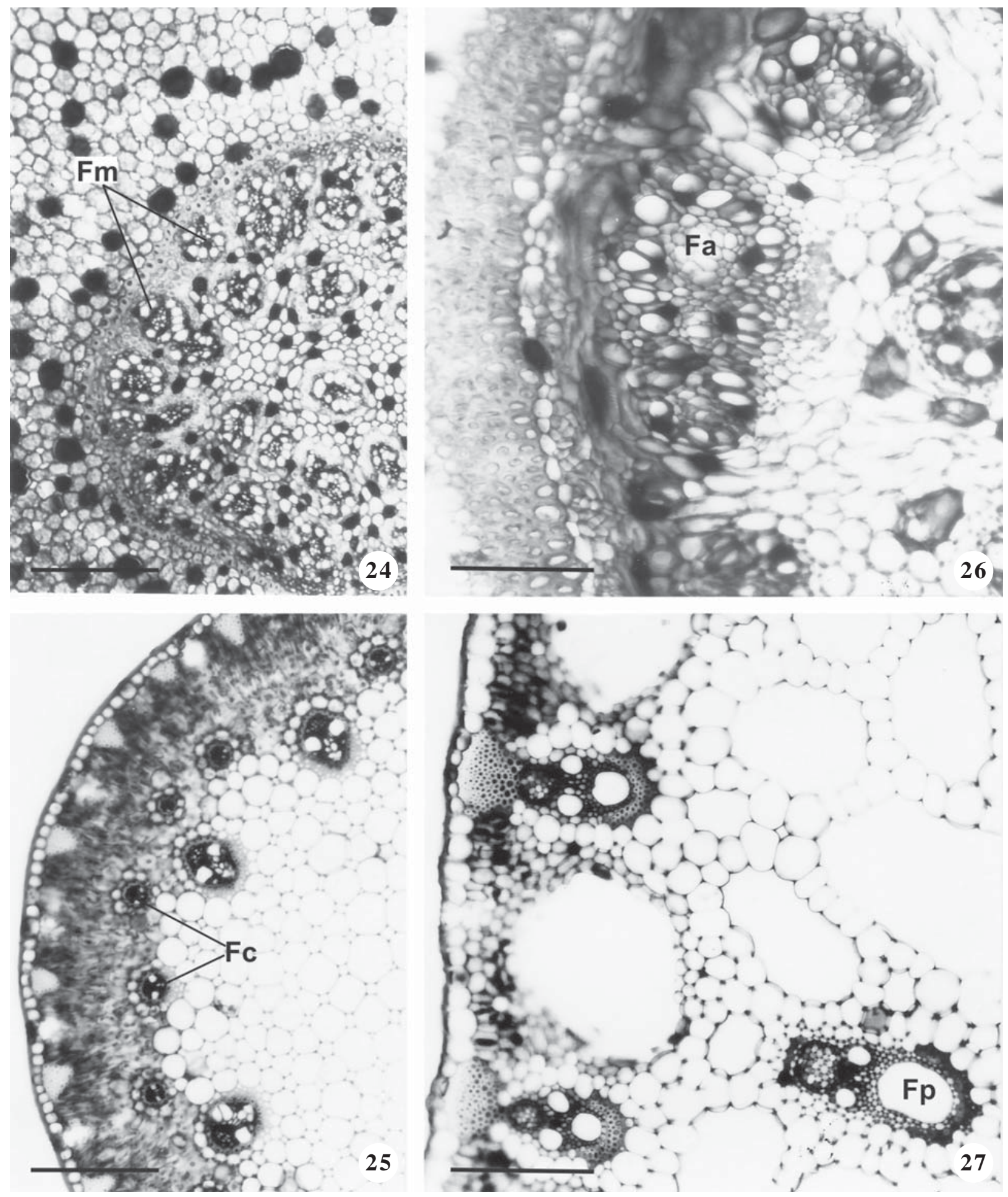

Figuras 24-27. Secções transversais de espécies de Cyperaceae. 24-25. Killinga peruviana. 26-27. Fimbrisylis autunnalis. 24, 26. Rizomas mostrando feixes anfivasais derivados do meristema de espessamento primário (MEP). 25, 27 Escapos mostrando feixes colaterais derivados do procâmbio $(\mathrm{Fa}=$ feixe anfivasal; $\mathrm{Fc}=$ feixe colateral; $\mathrm{Fp}=$ feixe procambial; $\mathrm{Fm}=$ feixe proveniente do MEP). Barras $=200 \mu \mathrm{m}(24,25), 100 \mu \mathrm{m}(26,27)$.

Figures 24-27. Transversal sections of the Cyperaceae species. 24-25. Killinga peruviana. 26-27. Fimbrisylis autunnalis. 24, 26. Rhizomes showing amphivasal bundles originated by the primary thickening meristem (MEP). 25, 27. Scapes showing colateral bundles originated of the procambium. $(\mathrm{Fa}=$ amphivasal bundle; $\mathrm{Fc}=$ colateral bundle; $\mathrm{Fp}=$ procambium bundle; $\mathrm{FM}$ $=$ vascular bundle from MEP). Bars $=200 \mu \mathrm{m}(24,25), 100 \mu \mathrm{m}(26,27)$. 
vasculares são todos colaterais, tipo considerado mais simples quando comparados com os anfivasais do rizoma de Cyperus (Mani 1963) e pelas autoras do presente estudo (dados não publicados).

Agradecimentos - As autoras agradecem ao Conselho Nacional de Desenvolvimento Científico e Tecnológico $(\mathrm{CNPq})$ e à Fundação de Amparo à Pesquisa do Estado de São Paulo (FAPESP) por auxílios financeiros.

\section{Referências}

ALONSO, A.A. \& MORAES-DALLAQUA, M.M. 2004. Morfoanatomia do sistema caulinar de Canna edulis Kerr-Gawler (Cannaceae). Revista Brasileira de Botânica 27:229-239.

BRUHL, J.B. \& PERRY, S. 1995. Photosynthetic pathway-related ultrastructure of $\mathrm{C}_{3}, \mathrm{C}_{4}$ and $\mathrm{C}_{3}$ like $\mathrm{C}_{3}-\mathrm{C}_{4}$ intermediate sedges (Cyperaceae), with special reference to Eleocharis. Australian Journal of Plant Physiology 22:521-530.

BUKATSCH, F. 1972. Bemerkungen zur Doppelfarbung Astrablau-Safranin. Mikrokosmos 6:225.

DAHLGREN, R.M.T., CLIFFORD, H.T. \& YEO, P.F. 1985. The families of the Monocotyledons. Structure, evolution and taxonomy. Berlin, Springer-Verlag.

DE MASON, D.A. 1979a. Function and development of the primary thickening meristem in the monocotyledon Allium cepa L. Botanical Gazette 140:51-66.

DE MASON, D.A. 1979b. Histochemistry of the primary thickening meristem in the vegetative stem of Allium cepa L. American Journal of Botany 66:347-350.

EAMES, A.J. 1961. Morphology of the angiosperms. McGrawHill, New York.

ESAU, K. 1965. Plant anatomy. John Wiley \& Sons, New York.

ESTELITA, M.E.M. 1993. Anatomia dos órgãos vegetativos de Remirea maritima Aubl. (Cyperaceae). Naturalia 18:123-134.

ESTELITA-TEIXEIRA, M.E. \& HANDRO, W. 1987. Kranz pattern in leaf, scape and bract of Cyperus and Fimbristylis species. Revista Brasileira de Botânica 10:105-111.

EVANS, P.S. 1965. Intercalary growth in the aerial shoot of Eleocharis acuta R. Br. I Structure of the growing zone. Annals of Botany 29:205-217.

FONT-QUER, P. 1975. Diccionário de botánica. 5ª ed. Editorial Labor, Barcelona.

GUAGLIANONE, R.E., UENO, O.\& RUTHSATZ, B. 1998. Notes on Eleocharis tucumanensis (Cyperaceae). Darwiniana 35:169-173.

JOHANSEN, D.A. 1940. Plant microtechnique. McGraw-Hill, New York.

KUKKONEN, I. 1967. Vegetative anatomy of Uncinia (Cyperaceae). Annals of Botany 31:523-544.

MANI, A.P. 1963.Types of vascular bundles in Cyperus and their possible trend of specialization. Science and Culture 29:37-39.
MENEZES, N.L., SILVA, D.C., ARRUDA, R.C.O., MELO-DEPINNA, G.F., CARDOSO, V.A., CASTRO, N.M., SCATENA, V.L. \& SCREMIN-DIAS, E. 2005. Meristematic activity of the endodermis and the pericycle in the primary tickening in monocotyledons. Considerations on the "PTM". Anais da Academia Brasileira de Ciências 77:259-274.

METCALFE, C.R. 1971. Anatomy of the monocotyledons. Cyperaceae. University Press, Oxford. v.5.

MUNRO, S.L. \& LINDER, H.P. 1998. A morphological-anatomy classification of organs in Monocots of diverse habit. In Second International Conference on the Comparative Biology of the Monocotiledons and Third International Symposium on Grass Systematics and Evolution (S.W.L. Jacobs \& J. Everett ed.). CSIRO, Sydney.

OTENG-YEBOAH, A.A. 1975. Morphology, anatomy and taxonomy of the genus Remirea Aubl. (Cyperaceae). Boissiera 24:197-205.

RODRIGUES, A.C. \& ESTELITA, M.E.M. 2002. Primary and secondary development of Cyperus giganteus Vahl. rizome (Cyperaceae). Revista Brasileira de Botânica 25:251-258.

RUDALL, P. 1984. Taxonomic and evolutionary implications of rhizome structure and secondary thickening in Iridaceae. Botanical Gazete 145:524-534.

SACHS, R.M. 1968. Control of intercalary growth in the scape of Gerbera by auxin and gibberellic acid. American Journal of Botany 55:62-68.

SEGECIN, S. \& SCATENA, V.L. 2004. Anatomia de escapos de Tillandsia L. (Bromeliaceae) dos Campos Gerais do Paraná, Brasil. Revista Brasileira de Botânica 27:515-525.

SHEPHERD, G.J. 1976. The use of anatomical characters in the intrageneric classification of Carex (Cyperaceae). Hoehnea 6:33-54.

SIMÃO, D.G. \& SCATENA, V.L. 2001. Morphology and anatomy in Heliconia angusta Vell. and $H$. velloziana L. Emygd. (Zingiberales: Heliconiaceae) from the Atlantic forest of Southeastern Brazil. Revista Brasileira de Botânica 24: 415-424.

SIMPSON, D.A. 1993. New species and a new combination in Cyperaceae from Brazil. Notes on Brazilian Cyperaceae VI. Kew Bulletin 48:699-713.

STEVENSON, D.W. \& FISHER, E. 1980. Radial growth in the Cycadales. American Journal of Botany 67:465-475.

TOMLINSON, P.B. 1970. Monocotyledons - towards and understanding of their morphology and anatomy. Advances in Botanical Research 3:207-292.

UENO, O., SAMEJIMA, M. \& KOYAMA, T. 1989. Distribution and evolution of $\mathrm{C}_{4}$ syndrome in Eleocharis, a sedge group inhabiting wet and aquatic environments, based on culm anatomy and carbon isotope ratios. Annals of Botany 64:425-438.

WILLS, G.D. \& BRISCOE, G.A. 1970. Anatomy of purple nutsedge. Weed Science 18:631-635.

ZIMMERMANN, M.H. \& TOMLINSON, P.B. 1969. The vascular system in the axis of Dracaena fragrans (Agavaceae). 1. Distribution and development of primary strands. Journal of the Arnold Arboretum 50:370-383. 